Association for Information Systems AIS Electronic Library (AISeL)

Wirtschaftsinformatik Proceedings 2001

Wirtschaftsinformatik

September 2001

\title{
Wissensmanagementprozesse - Mit Experteninterviews zum aufgabenbezogenen hypertextbasierten Informations- und Tutorsystem
}

Barbara Grille

FORWISS - Bayerisches Forschungszentrum für Wissensbasierte Systeme, grille@forwiss.de

Herbert Stoyan

FORWISS - Bayerisches Forschungszentrum für Wissensbasierte Systeme, stoyan@forwiss.de

Bernd Gaede

FORWISS - Bayerisches Forschungszentrum für Wissensbasierte Systeme, gaede@forwiss.de

Michael Müller

FORWISS - Bayerisches Forschungszentrum für Wissensbasierte Systeme, mlmuelle@forwiss.de

Follow this and additional works at: http://aisel.aisnet.org/wi2001

\section{Recommended Citation}

Grille, Barbara; Stoyan, Herbert; Gaede, Bernd; and Müller, Michael, "Wissensmanagementprozesse - Mit Experteninterviews zum aufgabenbezogenen hypertextbasierten Informations- und Tutorsystem" (2001). Wirtschaftsinformatik Proceedings 2001. 25.

http://aisel.aisnet.org/wi2001/25

This material is brought to you by the Wirtschaftsinformatik at AIS Electronic Library (AISeL). It has been accepted for inclusion in Wirtschaftsinformatik Proceedings 2001 by an authorized administrator of AIS Electronic Library (AISeL). For more information, please contact elibrary@aisnet.org. 
In: Buhl, Hans Ulrich, u.a. (Hg.) 2001. Information Age Economy; 5. Internationale Tagung Wirtschaftsinformatik 2001. Heidelberg: Physica-Verlag

ISBN: 3-7908-1427-X

(C) Physica-Verlag Heidelberg 2001 


\title{
Wissensmanagementprozesse - Mit Experteninterviews zum aufgabenbezogenen hypertextbasierten Informations- und Tutorsystem
}

\author{
Barbara Grille, Herbert Stoyan, Bernd Gaede, Michael Müller \\ FORWISS - Bayerisches Forschungszentrum für Wissensbasierte Systeme \\ Forschungsgruppe Wissenserwerb
}

Zusammenfassung: Seit einiger Zeit verfolgen wir den Ansatz, auf der Basis von Experteninterviews effizient Informations- und Tutorsysteme aufzubauen, in welchen sowohl Grundlagen als auch Handlungswissen dargestellt werden soll. Die zum Informationssystem komplementäre Entwicklung der Tutorsysteme mit einem CMS erlaubt die flexible Wiederverwendung beliebiger Informations- einheiten, die in verschiedenen didaktischen Rollen genutzt werden können.

Schlüsselworte: Wissensmanagement, Experteninterviews, Informations- und Tutorsysteme, aufgabenbezogene Modellierung, Wissensstrukturierung

\section{Einleitung}

Angesichts steigender Personalfluktuation und kürzerer Produktzyklen wird es für Unternehmen immer wichtiger, das Wissen erfahrener Experten zu sichern und zu verteilen sowie anderen Mitarbeitern so zugänglich zu machen, dass diese ihre Aufgaben schneller und besser bearbeiten können.

Bislang wurde Expertenwissen entweder mit arbeitsorganisatorischen Maßnahmen wie Gesprächen verteilt, oder man versuchte, dieses Wissen in Expertensystemen zu sichern. Während bei der ersten Form der Wissenssicherung das Wissen an Personen gebunden bleibt, sind bisherige Expertensysteme nicht geeignet, den sehr großen Teil kaum formalisierbaren Wissens zu repräsentieren. So begann man, Expertenwissen zu erheben und als Informationssystem (IS) in Hypertextform für das Intranet aufzubereiten. Die Nutzung dieses Wissens ist jedoch meist auf navigierenden und deskriptiven Zugriff beschränkt. Die Potenziale dieses Wissens für die Aus- und Weiterbildung von Mitarbeitern werden bislang noch nicht ausgeschöpft, da die Erstellung entsprechender Tutorsysteme sehr aufwendig ist. 
Wie [MaRe97] zeigen, beschränkt sich die Nutzung von Lernsoftware in der innerbetrieblichen Weiterbildung daher zumeist auf die Vermittlung allgemeiner Kenntnisse und wird nur selten auf firmenspezifische Inhalte ausgedehnt. Die auch informationstechnische Gestaltung betrieblicher Aus- und Weiterbildungsmaßnahmen hat nach [DeEr98] unter dem Schlagwort „lernende Unternehmen“ allerdings wieder vermehrte Aufmerksamkeit gefunden. [Ship96] führt aus, dass die Verwendung hypertextueller Wissenssysteme in Tutorsystemen z.B. im Bereich der Web Based Instruction seit langem thematisiert wird, wo heterogenes Wissen im WWW durch Einbettung in kohäsive Strukturen für das Lernen verwendbar gemacht werden soll. Didaktische Aspekte bei der Gestaltung von Tutorsystemen beschreiben u.a. [Blum98], [RiHo97] und [Schu97].

In diesem Artikel stellen wir die AbITus-Methodik vor, mit der im Rahmen des Wissensmanagements mit Experten aufgebaute Wissensbanken auf effiziente und didaktisch sinnvolle Weise für die interne Weiterbildung nutzbar gemacht werden. Im Einzelnen liefern wir die folgenden Beiträge zur effizienten Lösung des obigen Problems:

- Toolgestützte Erhebung des Expertenwissens mit strukturierten Interviews und eine durchgängige Methodik für den gesamten Workflow zur Erstellung von Tutorsystemen

- Verwendung von Templates und Guidelines zur strukturierten Wissenserhebung für Tutorsysteme, didaktische Referenzmodelle für die Vermittlung von Wissen über bestimmte Objekte, z.B. Bauteile oder Projektschritte, und von Wissen in bestimmten Fachgebieten, z.B. Automobilbau oder Marketing

- Wiederverwendung von Wissensstrukturen, z.B. als Defaults für Lerneinheiten, und von Wissensinhalten, z.B. Best Practices aus dem Informationssystem für Tutorsysteme

- Wissensbasierte Generierung von Tests in Tutorsystemen auf der Basis von Metainformationen und typisierten Links im IS, Generierung von Tutorsystemen in HTML aus medienneutral gehaltenen Wissensinhalten in einem Content-Management-System

Im folgenden Kapitel 2 wird die Kernidee der AbITus-Methodik eingeführt. Anschließend wird in Kapitel 3 deren praktische Umsetzung mit der Hilfe kommerzieller Content-Management-Systeme beschrieben, wobei auf Informationssysteme, aufgabenbezogene Tutorsysteme und den intendierten Transformationsprozess näher eingegangen wird. Das Kapitel 4 ist unseren Erfahrungen in der Praxis gewidmet. Dazu wird ein Beispiel vorgestellt, anhand dessen Hinweise auf wichtige Aspekte und mögliche Fehler bei der Umsetzung gegeben werden. Ein zusammenfassendes Fazit und ein Ausblick auf unsere weitere Arbeit in Kapitel 5 schließen diesen Artikel ab. 


\section{Die AbITus-Methodik}

Unser Ziel im AbITus-Projekt ist die effiziente Erstellung von Tutorsystemen. Die mit diesen Systemen erstellten Tutorien sollen Mitarbeiter, die mit neuen Aufgaben konfrontiert sind, anleiten.

Bisherige Ansätze zum Wissensmanagement konzentrieren sich häufig auf die Entwicklung von hypertextuellen Wissenssystemen. Hierbei besteht jedoch die Gefahr, dass ungeschulte Benutzer auf diese nicht zugreifen, da deren erfolgreiche Bedienung u.U. bereits ein tiefes Verständnis der Systemstrukturen und -inhalte erfordern könnte. Neue Mitarbeiter finden so möglicherweise nicht die für sie gerade relevante Information in der Sammlung von Expertenwissen. Diese Überlegung hat dazu geführt, dass wir einen hybriden Ansatz verfolgen, bei dem Tutorsysteme diese wichtigsten Informationen aufgabenbezogen in Tutorien darstellen und gleichzeitig die Orientierung im und den Umgang mit dem IS trainieren. Die Grundlagen dieses Ansatzes sind einerseits die klare Trennung zwischen Informations- und Tutorsystem, andererseits der aufgabenbezogene Entwurf von Tutorsystemen und die bedarfsgerechte Verlinkung beider Systeme. Die didaktische Basis der AbITus-Methodik ist die aufgabenbezogene Gestaltung von Tutorsystemen, die sich an Prinzipien der Anchored Instruction, des problemorientierten Lernens und des Situated Learning orientiert, deren praktische Anwendung zu erhöhter Motivation und größeren Lernerfolgen geführt hat.

Anhand der in [Brow98] beschriebenen Verfahren zur arbeitswissenschaftlichen Aufgabenanalyse wurde eine Struktur zur Beschreibung von Aufgaben und entsprechend vorbereiteten Curricula erarbeitet. Zur Anwendung kam eine vereinfachte Version des DACUM-Verfahrens (Develop A CUrriculuM), das in einer hierarchischen Stellen- bzw. Aufgabenbeschreibung resultiert. Die gemachten Einschränkungen beziehen sich auf die Anzahl beteiligter Experten und das interview- statt workshopbasierte Vorgehen. DACUM stützt sich auf drei Annahmen, denen wir uns im Projektszenario anschließen:

Experten können ihre Tätigkeit oder Aufgaben besser und genauer als sonst jemand beschreiben oder definieren.

Eine Stelle kann effektiv beschrieben werden, indem umrissen wird, welche Aufgaben ein Experte wie löst.

Alle Aufgaben erfordern zu ihrer ordentlichen Erledigung Wissen, Fähigkeiten, Werkzeuge und positive Anleitung des Ausführenden.

Diese Aufzählung adaptieren wir im Kontext von Tutorsystemen durch die zusätzliche Annahme, dass Experten durch eine tutoriell ausgerichtete Interviewführung bereits für die Erstellung des IS didaktisch relevantes Wissen und Hinweise formulieren. Außerdem beschränken wir uns inhaltlich auf notwendiges Wissen, Werkzeuge und die Anleitung. 
Eine resultierende Aufgabenbeschreibung bildet dann das strukturelle Gerüst für ein Tutorium, das durch ein realistisches Beispiel mit Leben gefüllt wird. Die Struktur wird als Gliederung verwendet, die mit einem Advanced Organizer nach Ausubel [Ausu60] ergänzt wird, der Lernziele des Tutoriums expliziert. Für jeden Teilschritt wird anschließend eine eigene Lerneinheit angelegt, durch die der Lerner bei der Bearbeitung des Beispiels angeleitet wird. Solchen Lerneinheiten werden anschließend differenziert nach didaktischen Funktionen relevante Inhalte aus dem IS zugeordnet. Diese bedarfsgerechte Zuordnung, z.B. als „Beispiel“ oder „Typischer Fehler“, weist referenzierten Inhalten aus dem IS eine Bedeutung im Kontext der aktuell erlernten Aufgabe zu. Dadurch werden die Informationen völlig unverändert in Tutorsysteme eingebunden, um die vielfältigen Ressourcen aus dem IS für unterschiedliche tutorielle Zwecke und Aufgaben nutzen zu können.

Da das IS auch als Wissensbasis für das Know-how der Abteilung fungiert, sollen alle Mitarbeiter mit ihm vertraut sein. Tutorsysteme ergänzen das ursprüngliche IS und motivieren Lerner zu dessen immer selbstständigerer Benutzung. Lerninhalte sind also Aufgaben der Mitarbeiter innerhalb von Projektabläufen. Gelernt wird anhand eines Beispiels, wie und mit welchen Mitteln man konkrete Aufgaben in der Abteilung bearbeitet. Die Entwicklungsqualität und -effizienz wird gesteigert durch:

- Guidelines für die didaktische Gestaltung der Lehrtexte in Anlehnung an [ThHo97] u. a.

- Templates für die Strukturierung der Kurse und Kursinhalte sowie

- die abschließende, vollautomatische Generierung der Hypertexte unter Beachtung ergonomischer und mediendidaktischer Aspekte.

Das Zusammenwirken dieser Aspekte wird im folgenden Kapitel näher beschrieben.

\section{Umsetzung der AbITus-Methodik mit einem Content-Management-System}

Die AbITus-Methodik kann unter ganz unterschiedlichen Voraussetzungen und für verschiedenste Domänen angewendet werden. So ist es grundsätzlich möglich, beliebig strukturierte Informationssysteme als Basis für die Tutorsystementwicklung zu nutzen. Um unsere Ausführungen übersichtlich zu halten, beschreiben wir im folgenden den Idealfall der parallelen Entwicklung des Informations- und Tutorsystems mit einem Content-Management-System (CMS) und weisen auf wesentliche Unterschiede zu anderen Szenarien hin. Unter einem CMS verstehen wir nach [Kart98] ein System, mit dem „Inhalte gespeichert, wiedergefunden, aufbereitet, aktualisiert, verwaltet und dann auf verschiedene Weise ausgegeben werden 
können, wobei die zusätzlichen Kosten für jede weitere Änderung und Ausgabe über die Zeit stark sinken“. Ein CMS bietet durch die Trennung von Inhalten, Struktur und Layout insbesondere die Möglichkeit, Inhaltsknoten oder Teilstrukturen in verschiedenen Kontexten wiederzuverwenden. CM-Systeme, die Hypertext als Ausgabeformat zulassen, verwalten Inhalte üblicherweise in Knoten, deren Beziehungen über Links dargestellt werden.

\subsection{Aufgabenbezogene Modellierung von Expertenwissen mit einem CMS}

Expertenwissen ist anders strukturiert als das Wissen von Laien [Brow97]. Um daraus resultierende Schwierigkeiten neuer Mitarbeiter bei der Informationssuche zu umgehen, erfassen wir Expertenwissen anhand konkreter Aufgaben aus dem Arbeitsalltag. Diese Form der Erhebung und Modellierung von Expertenwissen basiert auf der arbeitswissenschaftlichen Aufgabenanalyse und eigenen Erkenntnissen aus Erfahrungen in Wissenserwerbsprojekten.

Im CMS werden daher für die Modellierung des IS die folgenden Knoten- und Unterknotentypen zur Beschreibung von Aspekten jeder einzelnen Teilaufgabe zur Verfügung gestellt:

Allgemeines: Ziel, Inhalt und Gegenstand der Aufgabe

Prozess: Vorhergehende, Teil- und nachfolgende Schritte, Zeitrestriktionen

Wissen: Benötigtes Vorwissen, weitergehende und erklärende Informationen

Dokumente: Benötigte und zu erstellende Dokumente

Erfahrungen: Wichtige Hinweise, Best Practices, typische Fehler, positive und negative Beispiele

Werkzeuge: Methoden und Softwaretools

Personen: Rollen Beteiligter, Ansprechpartner

Diese Liste wird in konkreten Projekten meist noch geringfügig domänenspezifisch angepaßt, z.B. wenn keine Software verwendet wird. Die Modellierung einer Aufgabe mündet dann in eine hierarchische Repräsentation, wobei jeder Knoten einer Teilaufgabe entspricht, für welche die entsprechenden Informationen vom Experten erhoben werden.

Bereits existierende komplexe Hypertextinformationssysteme (z.B. externe Standardwerke in elektronischer Form) können für die Verwendung in Tutorsystemen entweder in das CMS importiert und restrukturiert oder über Stellvertreterknoten und externe Links integriert werden. Bei der letzten Variante sollten die aufgabenbezogenen Aspekte der extern bleibenden Informationen als Metadaten mit den Stellvertreterknoten verwaltet werden. Dann können auch solche Informationen zukünftig automatisch wiederverwendet werden. 


\subsection{Modellierung aufgabenbezogener Tutorsysteme mit einem CMS}

Wir unterscheiden bei der Modellierung aufgabenbezogener Tutorsysteme drei Templates, die den üblichen Phasen bei der Tutorsystemnutzung entsprechen. Mit einem $\uparrow$ markierte Punkte stellen Linktypen für Verweise dar.

Das Template für den Einführungsknoten ist wie folgt strukturiert:

Titel: für die schnelle Identifikation und Wiedererkennung des Tutorsystems

Media: zur Integration mediendidaktisch sinnvoller multimedialer Elemente (Eye Catcher)

Lernziele: Aktivierung und Orientierung der Lerner durch Explizierung des in diesem Schritt Lernbaren

Aufgabenrelevanz: Kontextualisierung durch Herstellen der Bezüge zwischen Lerninhalten und den Aufgaben des Lerners im Projektablauf; Motivation der Tutorsystembenutzung

个Voraussetzungen: Verlinkung vorher zu bearbeitender Tutorsysteme oder Informationen zur Orientierung der Lerner, um Frustration zu vermeiden und die Einordnung neuer Inhalte zu unterstützen

Zusammenfassung: als Übersicht für den eiligen Leser

个Lerneinheiten: Verlinkung der tutoriellen Knoten für Teilaufgaben und der Hilfeknoten zur Systembedienung

Die geschätzte Bearbeitungsdauer für die Nutzung des Tutorsystems wird zusammen mit anderen Informationen wie der Relevanz, Erstellungs- und Änderungsdatum oder Autor in der Form von Metadaten verwaltet. In der Literatur findet man darüber hinaus Mengen vorgeschlagener Metadaten für Lernmaterialien, die teilweise sogar standardisiert sind. Interessant wäre an diesen Ansätzen in unserem Zusammenhang insbesondere die Beschreibung der didaktischen Funktion der Lernmaterialien, für die aber leider noch keine ausgearbeiteten Standards existieren.

Das Template für den Präsentationsknoten ist folgendermaßen untergliedert, wobei Verweise ( $\uparrow$ ) ins IS führen:

Name: dient wiederum der schnellen Identifikation und Wiedererkennung des Teilschritts

Anleitung: gruppiert den wesentlichen, beispielhaften Part des Tutoriums Stand im Beispiel, Beispielschritt ausführen, Ergebnis im Beispiel

Wissen: gruppiert alle nützlichen Informationen für die Bearbeitung eines Teilschritts

$\uparrow$ Wichtige Hinweise, $\uparrow_{\text {Best }}$ Practices, $\uparrow$ Typische Fehler, $\uparrow$ Ansprechpartner, $\uparrow$ Rollen Beteiligter, $\uparrow$ Begriffsdefinitionen (Verweise in ein Glossar), $\uparrow$ Weitergehende und erklärende Informationen

Hilfsmittel: verweist auf Methoden und Werkzeuge oder andere relevante Utensilien 
Richtlinien, $\uparrow$ Formulare/Formatvorlagen für zu erstellende Dokumente, $\uparrow$ Methoden, $\uparrow$ Software

Der Anleitungsteil liefert dabei den essentiellen Mehrwert des Tutorsystems. Die Untergliederung dieses Punktes wird von uns nur durch redaktionelle Hinweise erreicht, um die Formulierung des Beispieltextes nicht zu schablonenhaft werden zu lassen. Alle weiteren Aspekte (Wissen, Hilfsmittel) werden durch die bloße Verlinkung mit Inhalten im IS berücksichtigt. Um den Lernern und Nutzern die Orientierung im IS zu erleichtern, sollten abteilungstypische Unterscheidungen von Informationen (Vorschrift, Richtlinie, Beispiel, Ausnahme, Zeichnung usw.) kenntlich gemacht und dazu in den Metadaten mitgeführt werden. Aus pragmatischen Gründen beschränken wir uns gegenwärtig auf die wichtigsten und einfach zu bestimmende Metadaten wie die Bearbeitungsdauer und eine ontologische Attributierung, die den Automatisierungsgrad der Tutorsystemproduktion erhöhen kann.

Templates für verschiedene Tests werden anhand der Strukturinformationen im IS automatisch befüllt. Auf diese Weise befüllte Aufgabenschablonen bieten Testfragen zur Erfolgskontrolle für die Lerner.

\subsection{Vom Informations- zum Tutorsystem}

Im Idealfall sollen beide Systeme, also das IS und das Tutorsystem (TS), von dem Wissensingenieur während des Experteninterviews befüllt werden. $\mathrm{Zu}$ diesem Zweck wird das Interview mit bewährten Fragebögen geführt, die zunächst die strukturierte Erhebung aller oben beschriebenen Inhalte für das IS unterstützen. Eine solche abgeschlossene Aufgabenbeschreibung im IS wird dann analog mit TS-Knoten nachgebildet, deren Anleitungsteil anhand eines durchgehenden Beispiels befüllt wird. Sind die IS-Knoten zuvor nicht ausreichend befüllt worden, können sie auch nicht sinnvoll den einzelnen TS-Knoten (Einführungsknoten, Präsentationsknoten sowie Testknoten) zugeordnet werden, so dass dieser Klärungsbedarf bei der sofortigen Verlinkung zwischen TS und IS unverzüglich berücksichtigt werden kann.

Durch die gleichzeitige Beachtung beider Systemstrukturen während des Interviews soll gewährleistet werden, dass auf alle für einen Vorgang relevanten Informationen zugegriffen werden kann. Bisherige Erfahrungen haben gezeigt, dass bei der nachträglichen Gestaltung konkreter TS-Beispiele wichtige Informationen im IS fehlten, da deren Relevanz im Rahmen der bisherigen Vorgehensweise nicht erkannt und entsprechende IS-Knoten nicht erstellt wurden.

Entkoppelt vom Interview ist es möglich, Verknüpfungen von standardmäßig korrelierten IS- und TS-Knoten automatisch herzustellen. Die Verknüpfung zwischen den Unterknoten der Kategorie „Hilfsmittel“ im TS-Knoten mit den entsprechen- 
den Knoten „Werkzeuge, Software“ und „Richtlinien, Formulare, Dokumente“ im IS ist ein Beispiel dafür.

Um Schwierigkeiten und Fehlern neuer Mitarbeiter vorzubeugen, sollte deren Vermeidung trainiert werden. Zu diesem Zweck kann es sinnvoll sein, zusätzlich zu den unten beschriebenen generierten Tests auch manuell Tests im CMS zu modellieren. Beispielsweise sollten die Inhalte im Informationsknoten „Wichtige Hinweise" mit Hilfe adäquater Aufgabentypen für Trainingszwecke aufbereitet werden. Die entsprechende „Musterlösung“ sollte sich in den Inhalten des ISKnotens wiederfinden, wodurch eine manuelle Verlinkung möglich wird, die idealiter während des Interviews durch den Experten abgesichert erfolgen sollte.

Die Effizienz bei der Erstellung der Tutorsysteme wird durch unterschiedliche Mechanismen gesteigert. Die Unterstützung reicht dabei von Qualitätsverbesserungen durch die Angabe von didaktisch motivierten Redaktionsrichtlinien (Guidelines) über die Strukturierungshilfe durch Knoten- und Linktypen bis hin zur Automatisierung der Verlinkung und der Generierung von Tests für die Lernfortschrittskontrolle und von hypermedialen Tutorsystemen.

Die Vorgabe arbeitswissenschaftlich oder didaktisch fundierter Knotenstrukturen und Links unterstützt den Wissensingenieur bei der vollständigen Erhebung relevanter Informationen und führt gewissermaßen zur Wiederverwendung des entsprechenden arbeitswissenschaftlichen oder didaktischen Know-how. Das entkoppelte Layout erspart die Formatierung neuer Inhalte und kann deren konsistente und ergonomische Darstellung im Informations- oder Tutorsystem gewährleisten. Gerade in Lernsituationen ist dies wichtig, um Verwirrungen (z.B. durch eine nicht erkennbare Gliederung) und das Phänomen der kognitiven Überlastung (z.B. durch Ablenkung vom Lernstoff wegen Schwierigkeiten bei Systembedienung) zu vermeiden.

Die automatische Verlinkung basiert ebenfalls auf der sorgfältigen Modellierung konkreter Aufgaben im IS. So kann ein Tutorsystemknoten zum Thema „Erstellen von Postern“ (s. Abschnitt 4.1) nur dann automatisch auf ein besonders gelungenes Poster verweisen, wenn dieses auch im IS als ,positives Beispiel“ geführt wird. In ähnlicher Weise ist auch die Zusammenfassung schwach befüllter Teilaufgaben denkbar, indem z.B. zwei Ansprechpartner für alle Teilaufgaben der Messevorbereitung direkt in diesem Knoten angezeigt werden. Die automatische Verlinkung kann für externe Informationssysteme ebenfalls erfolgen, indem diese Modellierung durch Metadaten und Stellvertreterknoten simuliert wird. Dieses relativ triviale Beispiel sollte nicht über den Nutzen der automatischen Verlinkung in komplexen Informationssystemen hinwegtäuschen.

Lernfortschrittskontrollen oder Lernerselbstkontrollen kommt in Tutorsystemen eine wichtige Rolle zu. Die Entwicklung entsprechender Mechanismen scheitert in der Regel aber an dem erforderlichen hohen Aufwand. Daher generieren wir Fragen soweit wie möglich automatisch. Auch hier ist die sorgfältige Modellierung von IS und TS die notwendige Voraussetzung, damit beispielsweise zum Knoten 
„Vorbereitung von Messeständen“ anhand ausgehender Links vom Typ „Teilaufgabe“ auf Knoten mit hoher Relevanz (Metadatum) die Frage nach „wichtigen Teilaufgaben im Rahmen der Vorbereitung von Messeständen“ generiert werden kann. Interessant ist dabei auch die teilweise mögliche Generierung automatischer Auswertungsmechanismen. Wo diese nicht möglich ist, können häufig noch Aufgaben generiert werden, die den Lerner zur Selbstkontrolle anhalten: „Füllen Sie das vorliegende Gesprächsprotokollformular zur Nachbereitung des Messebesuchs aus!" Statt einer Antwort könnte solchen einer Aufgabe dann gegebenenfalls immerhin ein besonders gut ausgefülltes Beispiel zum Vergleich mit der eigenen Lösung zur Seite gestellt werden. Für nicht generierbar halten wir Tests, die Transferaufgaben stellen oder sogar Problemlöseverhalten prüfen sollen.

\section{AbITus in der Praxis}

In diesem Kapitel veranschaulichen wir die bisherigen Ausführungen anhand eines konkreten Beispiels und fassen unsere bisherigen Erfahrungen mit der Anwendung der AbITus-Methodik zusammen.

\subsection{Ein Beispiel - Erstellung eines Tutorsystems für die Vorbereitung von Messeständen}

Hintergrund für das Beispiel war das bevorstehende Ausscheiden eines langjährigen Mitarbeiters aus der Forschungsgruppe Wissenserwerb des FORWISS. Sein Erfahrungswissen zu wichtigen Aufgaben sollte erhoben, in einem Content-Management-System gesichert und in Form eines Hypertexts und eines Tutoriums den Mitarbeitern zugänglich gemacht werden. Für das Beispiel wählten wir die Aufgabe „Vorbereitung von Messeständen“ als Teilaspekt des Bereichs “Öffentlichkeitsarbeit und Marketing" aus. Als Vorbereitung auf das Experteninterview stellte der Wissensingenieur aus Seminarunterlagen über Messestände eine initiale Aufgabenstruktur zusammen, wobei zu jeder Aufgabe die in Abschnitt 3.1 beschriebenen Aspekte geheftet wurden. Dieser Fragebogen war Grundlage des folgenden Experteninterviews, in dem zunächst mit dem Experten die Informationen für den Einführungsknoten des Tutorsystems erarbeitet wurden: 


\begin{tabular}{|c|c|}
\hline $\begin{array}{l}\text { Einführungsknoten } \\
\text { im Tutorsystem }\end{array}$ & Beispiel \\
\hline Titel & Vorbereitung von Messeständen \\
\hline Lernziele & $\begin{array}{l}\text { Nach diesem Kurs sollten Sie die einzelnen Arbeitsschritte, wichtige } \\
\text { Ansprechpartner, relevante Dokumente und wichtige Hinweise für die } \\
\text { Vorbereitung von Messeständen kennen. }\end{array}$ \\
\hline Aufgabenrelevanz & $\begin{array}{l}\text { Da sich unser Forschungsinstitut auch durch Drittmittelprojekte mit } \\
\text { Partnern aus der Industrie finanziert, sind Maßnahmen der Öffentlich- } \\
\text { keitsarbeit und des Marketing wichtig. Dazu gehören insbesondere } \\
\text { Messestände. } \\
\text { Sich über die Vorbereitung von Messeständen weiterzubilden, ist ins- } \\
\text { besondere deshalb wichtig, weil diese Phase in ihrer Bedeutung häufig } \\
\text { unterschätzt wird. Außerdem verursachen Messestände hohe Kosten, } \\
\text { und in der Vergangenheit sind immer wieder dieselben Fehler gemacht } \\
\text { worden. Mit dem vermittelten Wissen ist eine effizientere Vorberei- } \\
\text { tung möglich. }\end{array}$ \\
\hline Voraussetzungen & $\%$ \\
\hline Zusammenfassung & $\begin{array}{l}\text { Sie sind neuer Mitarbeiter in der Forschungsgruppe Wissenserwerb } \\
\text { und haben von ihrem Leiter Prof. St. den Auftrag erhalten, den Messe- } \\
\text { stand für die Fachausstellung der Systems } 2000 \text { vorzubereiten. ... }\end{array}$ \\
\hline Media & Photo eines Messestands \\
\hline Lerneinheiten & $\begin{array}{l}\text { Teilaufgaben bei der Vorbereitung von Messeständen, Auswahl und } \\
\text { Erstellung der Poster usw. }\end{array}$ \\
\hline
\end{tabular}

Tabelle 1: Einträge des Einführungsknotens im Tutorium

Der Experte wählte als durchgängige Story die Vorbereitung des Messestands für die Fachausstellung der Systems 2000, da er aktuell mit dieser beschäftigt war. Bei der Systems handelt es sich um eine anwendungsorientierte Kongressmesse. Zudem trat die Besonderheit auf, dass mehrere Vortragende auf der Tagung waren, ein Spin-off-Unternehmen sich als Partner beteiligen wollte, und neue Poster mit einer Werbeagentur zu erstellen waren. Danach wurde im Interview die initiale Aufgabenstruktur vom Experten korrigiert und vervollständigt bzw. auf die spezifische Situation in einem Forschungszentrum angepasst. Dabei wurde die Aufgabe hierarchisch in mehrere Teilaufgaben wie etwa ,Zusammenstellung des Messeteams“, „Festlegung der Aussteller“ und „Klärung der Finanzierung“ zerlegt. Zu diesen Teilaufgaben wurde vom Experten Wissen über Ansprechpartner, relevante Dokumente, Best Practices und wichtige Hinweise erhoben sowie die Story fortgesetzt: 


\begin{tabular}{|c|c|c|}
\hline $\begin{array}{l}\text { Präsentation- } \\
\text { knoten im Tu- } \\
\text { torsystem }\end{array}$ & $\begin{array}{l}\text { Aufgabenknoten } \\
\text { im Informati- } \\
\text { onssystem }\end{array}$ & Beispiel \\
\hline Titel & Titel & Zusammenstellung des Messeteams \\
\hline \multirow[t]{3}{*}{ Wissen } & Ansprechpartner & $\begin{array}{l}\text { Zwei Mitarbeiter der Gruppe, die für Marketing und Öf- } \\
\text { fentlichkeitsarbeit zuständig sind: Oliver H. und Harald } \\
\text { S. Sollten beide Ansprechpartner nicht zur Verfügung } \\
\text { stehen, kann man sich an den stellvertretenden FG-Leiter } \\
\text { Dr. Michael M. oder an den Assistenten der Geschäfts- } \\
\text { führung Gerd G. wenden. }\end{array}$ \\
\hline & Best Practices & $\begin{array}{l}\text { Es hat sich bei der Teambildung bewährt, folgenden } \\
\text { Funktionen bzw. Zuständigkeiten konkrete Personen } \\
\text { zuzuordnen: Projektleiter, Ersteller der Demo, Ersteller } \\
\text { der Poster und Prospekte, Ansprechpartner für die Orga- } \\
\text { nisation in der Geschäftsführung und Betreuer des Mes- } \\
\text { sestands. }\end{array}$ \\
\hline & Wichtige Hinweise & $\begin{array}{l}\text { Bei der Auswahl der Standbetreuer ist darauf zu achten, } \\
\text { dass diese neben der fachlichen Kompetenz auch über } \\
\text { folgende Fähigkeiten verfügen: gutes äußeres Auftreten, } \\
\text { kommunikative Fähigkeiten, Mehrsprachigkeit bei inter- } \\
\text { nationalen Messen, Überblick über das FORWISS usw. } \\
\text { Im Idealfall sollte ... }\end{array}$ \\
\hline Hilfsmittel & Software & $\begin{array}{l}\text { Mit } \uparrow \text { MS-Outlook können Sie die Funktionalität von } \\
\uparrow \text { MS-Exchange nutzen, um verfügbare Mitarbeiter zu } \\
\text { koordinieren! }\end{array}$ \\
\hline Anleitung & & $\begin{array}{l}\text { Stand im Beispiel: Sie haben den Auftrag erhalten, den } \\
\text { Messestand für die Fachausstellung der Systems } 2000 \\
\text { vorzubereiten. } \\
\text { Beispielschritt ausführen: Als erstes sollten Sie mit den } \\
\text { beiden zuständigen Mitarbeitern Kontakt aufnehmen. ... } \\
\text { Die bei der Tagung beteiligten Mitarbeiter kommen als } \\
\text { potentielle Betreuer des Messestands in Frage. ... } \\
\text { Ergebnis im Beispiel: Am Ende dieser Phase sollten alle } \\
\text { Zuständigkeiten verteilt und die Betroffenen informiert } \\
\text { sein. }\end{array}$ \\
\hline
\end{tabular}

Tabelle 2: Einträge eines Präsentationsknotens im Tutorium

Das Interview endete damit, dass der Experte nochmals die wichtigsten Teilaufgaben der Vorbereitung von Messeständen betonte. Dazu gehörten die Vorbereitung von Messeständen als komplexe Aufgabe, die Auswahl und Erstellung der Poster, die Planung und Durchführung der Marketingmaßnahmen, die Zusammen- 
stellung bzw. Reservierung der Messeutensilien sowie das Training bzw. Briefing der Kundenbetreuung. Darauf basierend wurde in dem Content-Management-System ein Tutorsystem mit den entsprechenden fünf Lerneinheiten angelegt.

Abbildung 1: Ausschnitt aus der Modellierung mit einem CMS

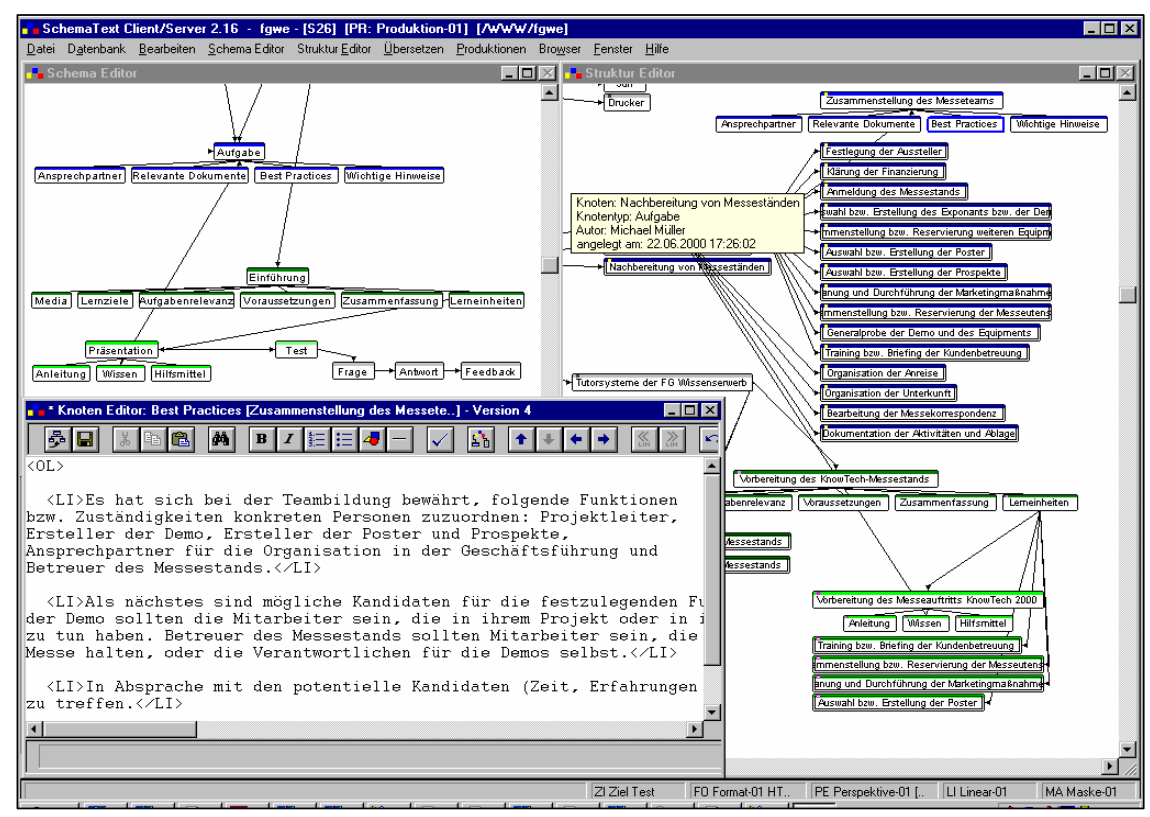

Bei der Generierung des Tutoriums aus diesen Inhalten können durch die oben beschriebenen Mechanismen die folgenden Tests zu den fünf Lerneinheiten generiert werden, um den Lernerfolg hinsichtlich der wichtigsten Lernziele zu überprüfen. Die Automatisierbarkeit der Erfolgskontrolle hängt dabei allerdings von der Modellierungsqualität und vom jeweiligen Aufgabentyp ab, wobei Freitextantworten im Gegensatz zu Auswahlfragen eine Automatisierung weitgehend verhindern: 


\begin{tabular}{|c|c|c|c|}
\hline Lerneinheit & $\begin{array}{l}\text { Automatisierbarkeit } \\
\text { der Erfolgskontrolle }\end{array}$ & Frage & $\begin{array}{l}\text { Antwort bzw. } \\
\text { Musterlösung } \\
\end{array}$ \\
\hline $\begin{array}{l}\text { Vorbereitung von } \\
\text { Messeständen }\end{array}$ & $\begin{array}{l}\text { kaum } \\
\text { automatisierbar }\end{array}$ & $\begin{array}{l}\text { Nennen Sie wich- } \\
\text { tige Teilaufgaben } \\
\text { im Rahmen der } \\
\text { Messevorbereitung! }\end{array}$ & $\begin{array}{l}\text { Selbstkontrolle durch Ge- } \\
\text { genüberstellung der gelie- } \\
\text { ferten Teilaufgaben mit } \\
\text { der vollständigen Liste }\end{array}$ \\
\hline $\begin{array}{l}\text { Auswahl und Er- } \\
\text { stellung der Poster }\end{array}$ & Automatisierbar & $\begin{array}{l}\text { Welche der vorlie- } \\
\text { genden Poster sind } \\
\text { für die vorliegende } \\
\text { Messe geeignet? }\end{array}$ & $\begin{array}{l}\text { Automatische Kontrolle } \\
\text { durch Vergleich der aus- } \\
\text { gewählten Poster mit den } \\
\text { positiven und negativen } \\
\text { Beispielen }\end{array}$ \\
\hline $\begin{array}{l}\text { Planung und Durch- } \\
\text { führung der Marke- } \\
\text { tingmaßnahmen }\end{array}$ & Automatisierbar & $\begin{array}{l}\text { Ordnen Sie die fol- } \\
\text { genden } \\
\text { Marketingmaß- } \\
\text { nahmen nach } \\
\text { Wichtigkeit! }\end{array}$ & $\begin{array}{l}\text { Automatische Kontrolle } \\
\text { durch Vergleich der gelie- } \\
\text { ferten Ordnung mit der } \\
\text { vorgegebenen Ordnung }\end{array}$ \\
\hline $\begin{array}{l}\text { Zusammenstellung } \\
\text { bzw. Reservierung } \\
\text { der Messeutensilien }\end{array}$ & $\begin{array}{l}\text { kaum } \\
\text { automatisierbar }\end{array}$ & $\begin{array}{l}\text { Nennen Sie wich- } \\
\text { tige Messeutensi- } \\
\text { lien? }\end{array}$ & $\begin{array}{l}\text { Selbstkontrolle durch Ge- } \\
\text { genüberstellung der gelie- } \\
\text { ferten Messeutensilien mit } \\
\text { der vollständigen Messe- } \\
\text { checkliste }\end{array}$ \\
\hline $\begin{array}{l}\text { Training bzw. Brie- } \\
\text { fing der Kundenbe- } \\
\text { treuung }\end{array}$ & $\begin{array}{l}\text { kaum } \\
\text { automatisierbar }\end{array}$ & $\begin{array}{l}\text { Füllen Sie das vor- } \\
\text { liegende Ge- } \\
\text { sprächsprotokoll } \\
\text { aus! }\end{array}$ & $\begin{array}{l}\text { Selbstkontrolle durch Ge- } \\
\text { genüberstellung des aus- } \\
\text { gefüllten Gesprächsproto- } \\
\text { kolls mit dem Muster ei- } \\
\text { nes Gesprächsprotokolls }\end{array}$ \\
\hline
\end{tabular}

Tabelle 3: Tests im Tutorium

\subsection{Erfahrungen mit der AbITus-Methodik}

Tutorsysteme können nach der AbITus-Methodik schneller als üblich entwickelt werden. Die Mikrostruktur, also die Untergliederung einzelner tutorieller Knoten, kann die Didaktisierung der Lerneinheiten fördern. Mit sehr feingranularen didaktischen Strukturvorgaben werden die Autoren allerdings zu stark eingeschränkt und die generierten Kurse in der Folge zu stereotyp. Daher verwenden wir statt der anfänglichen acht Unterknoten, die mit didaktischen Funktionen korreliert waren, nur noch vier Unterknoten, die Aspekte der arbeitswissenschaftlichen Aufgabenanalyse aufgreifen.

Didaktische Aspekte können von Fachexperten durch anleitende Guidelines flexibler und besser berücksichtigt werden. Wesentlich für die erfolgreiche Tutorsy- 
stemgestaltung ist aber die Auswahl der Lerninhalte bzw. der Beispiele, anhand derer Aufgaben erlernt werden sollen.

Für jede zu erlernende Aufgabe muss der Experte ein konkretes Beispiel hinreichend detailliert beschreiben. Die Konstruktion realistischer Beispiele aus dem IS ist für den Wissensingenieur in der Regel nicht möglich. Um Erfahrungen mit solchen Beispielen zu sammeln, sollte der Entwickler zunächst nur sehr kleine Aufgaben betrachten. Die Komplexität der Aufgaben kann durch Einschränkungen des Kontextes weiter reduziert werden. Beispielsweise könnte die Aufgabe „Bereiten Sie geeignete Exponate für die Messe vor!" vereinfacht werden, indem dazu benötigte Materialien bereits vorgegeben werden: „Ein Kollege hat Ihnen bereits die Poster zusammengestellt. Nun benötigen Sie noch Faltblätter!“ Bisher wurde das IS ohne Blick auf das geplante TS erstellt. Die Erfahrung hat jedoch gezeigt, dass für das TS relevante Informationen dadurch nicht erfragt worden sind, wodurch die effiziente Erstellung eines Tutorsystems zumindest behindert wurde. Sinnvoll ist daher, den Experten das Tutorsystem mit seinen Anleitungs- und Testmechanismen genau zu erläutern, da anzunehmen ist, dass die Informationsknoten zielgerichteter bzw. lernerorientierter befüllt werden.

\section{Zusammenfassung und Ausblick}

Um auf einfache Weise die intendierte Zielgruppe ansprechende Tutorsysteme produzieren zu können, entwickeln wir aufgabenorientierte Tutorsysteme, welche die Inhalte von Informationssystemen integrieren. Die zum Informationssystem komplementäre Entwicklung der Tutorsysteme mit einem CMS erlaubt die flexible Wiederverwendung beliebiger Inhaltsknoten, so dass ein solcher Knoten sogar in verschiedenen didaktischen Rollen genutzt werden kann. Auf diese Weise wird mit unserer Methodik die Entwicklung von Tutorsystemen nach den Prinzipien des aufgabenbezogenen Curriculumdesigns unterstützt.

Hier wurde das eigens entwickelte Vorgehensmodell für die tutorielle Nutzung von Informationssystemen vorgestellt. Um insbesondere neuen Mitarbeitern die relevantesten Inhalte didaktisch aufbereitet präsentieren zu können, wurde eine pragmatische Lösung entwickelt, mit der jedes strukturorientiert verwaltete Informationssystem unverzüglich um tutorielle Aspekte ergänzt werden kann. Unsere Methodik basiert aus Effizienzgründen auf Standardmechanismen moderner Content-Management-Systeme. So können mit minimiertem Aufwand motivierende Tutorials entwickelt werden, die im Sinne der Anchored Instruction „,bedeutungshaltige Problemlösungskontexte" für potentielle Lerner bereitstellen.

Erweiterungsmöglichkeiten für AbITus-Tutorsysteme gibt es in zweierlei Hinsicht: Zunächst erlaubt es der Einsatz von Standardsoftwarekomponenten, weitere pragmatische Ergänzungen vorzunehmen, z.B. die Integration von Foren, erwei- 
terbaren FAQ-Listen, Whiteboard-Techniken bis hin zu Application Sharing und Videokonferenzen. Sie erschließen ohne großen Aufwand neue Nutzungspotenziale einer Wissensbank. Hierbei handelt es sich jedoch um Enabling Technology, die wie die meisten Wissensmanagementtechnologien ohne begleitende Maßnahmen kaum Verwendung finden werden.

Aus pädagogischer Sicht lassen sich weitere Verbesserungen vorstellen. So hängt die effiziente Erstellung nicht nur von einem technisch funktionierenden System $\mathrm{ab}$, sondern auch von der genauen Kenntnis der pädagogischen Relationen. Unter pädagogischen Relationen sind hier die Beziehungen zwischen den einzelnen am Wissenstransfer beteiligten Personen zu verstehen.

Weitere mögliche pädagogische Verbesserungen sind die themenspezifische, persönliche Betreuung der Lerner durch Tutoren, die Integration multimedialer Komponenten (Videos, Animationen) zu Motivationszwecken, die Entwicklung weitergehender Übungsaufgaben, welche die Anwendung, Festigung und den Transfer des Gelernten erlauben, und die Bereitstellung alternativer Varianten von Online-Kursen für verschiedene Lernstilpräferenzen der Zielgruppe. Von großem Interesse sind auch empirische Studien über den Nutzen der Tutorsysteme, deren Ergebnisse zu weiteren Verbesserungsvorschlägen führen könnten.

Langfristig planen wir auch die ontologiebasierte Verschlagwortung aller Inhalte. Auf dieser Grundlage können dann weitere Nutzungsmöglichkeiten teilweise automatisiert unterstützt werden. Beispielsweise wird so die automatische Integration neuer Informationen in bestehende Tutorsysteme oder deren adaptive Konfiguration anhand spezifischer Vorkenntnisse und Anforderungen vorstellbar.

\section{Literatur}

[Ausu60] Ausubel, D.P.: The use of advanced organizers in the learning and retention of meaningful verbal material. Journal of Educational Psychology, (1960) 51, 267-272.

[Blum98] BLUMSTENGEL, A.: Entwicklung hypermedialer Lernsysteme, (http://dsor.uni-paderborn.de/organisation/blum_diss/main_index_titel.html), 1998, Abruf $8 / 2000$.

[Brow98] Brown, B.L.: Task Analysis Strategies and Practices, http://ericacve.org/docs/ pab00003.htm, 1998, Abruf 8/2000.

[Brow97b] Brown, T.: Multimedia in Education, http://scs.une.edu.au/Materials/ 573/573Menu, 1997, Abruf 8/2000.

[DeEr98] Dehnbostel, P., Erbe, H.-H., Novak, H.: Berufliche Bildung im lernenden Unternehmen. edition sigma, Berlin 1998. 
[Kart98] Kartchner, C.: Content Management Systems: Getting from Concept to Reality. The Journal of Electronic Publishing, 3 (4), (http://www.press.umich.edu/jep/03-04/), 1998, Abruf 8/2000.

[MaRe97] Mandl, H., Reinmann-Rothmeier, G.: Lernen mit Multimedia. Forschungsbericht 77 der Ludwig-Maximilians-Universität München, 1997.

[RiHo97] Ritchie, D., Hofman, B.: Incorporating instructional design principles with the world wide web. In: Khan B. ed. Web based instruction. NJ:Educational Technology Publications, 135-138, (http://www.gwu.edu/ etlalex/khan/tc.txt), 1997, Abruf 8/2000.

[Schu97] Schulmeister, R.: Grundlagen Hypermedialer Lernsysteme. Addison-Wesley, Berlin 1997.

[Ship96] Shipman, F. et al.: Creating Educational Guided Paths over the World Wide Web In Educational Telecommunications. In: P. Carlson, F. Makedon eds. Proceedings of ED-TELECOM 96, Bosten USA, 17 - 22 Juni 1996. Charlottesville 1996, S. 326-331.

[ThHo97] Thiel, J., Hoffman, B.: Document Preparation for Online Courses (http://edwebiii.sdsu.edu/t3/module2/intro.htm), 1997, Abruf 8/2000. 